\title{
Loss of ARID1A protein expression occurs as an early event in ovarian clear-cell carcinoma development and frequently coexists with PIK3CA mutations
}

\author{
Sohei Yamamoto ${ }^{1}$, Hitoshi Tsuda ${ }^{2}$, Masashi Takano ${ }^{3}$, Seiichi Tamai ${ }^{4}$ and \\ Osamu Matsubara ${ }^{1}$ \\ ${ }^{1}$ Department of Basic Pathology, National Defense Medical College, Tokorozawa, Japan; ${ }^{2}$ Department of \\ Pathology and Clinical Laboratories, National Cancer Center Hospital, Tokyo, Japan; ${ }^{3}$ Department of \\ Obstetrics and Gynecology, National Defense Medical College, Tokorozawa, Japan and ${ }^{4}$ Department of \\ Laboratory Medicine, National Defense Medical College Hospital, Tokorozawa, Japan
}

\begin{abstract}
$A R I D 1 A$ is a recently identified tumor suppressor gene that is mutated in $\sim 50 \%$ of ovarian clear-cell carcinomas. This mutation is associated with loss of ARID1A protein expression as assessed by immunohistochemistry. The present study aimed at determining the timing of the loss of ARID1A protein expression during the development of ovarian clear-cell carcinoma and assessing its relevance in correlation to PIK3CA gene mutations. A total of 42 clear-cell carcinoma cases with adjacent putative precursor lesions (endometriosis-associated carcinoma cases $(n=28)$ and (clear-cell) adenofibroma-associated carcinoma cases $(n=14))$ were selected and subjected to immunohistochemical analysis for ARID1A protein expression and direct genomic DNA sequencing of exons 9 and 20 of the PIK3CA gene. ARID1A immunoreactivity was deficient in $17(61 \%)$ of the 28 endometriosis-associated carcinomas and $6(43 \%)$ of the 14 adenofibroma-associated carcinomas. Among the precursor lesions adjacent to the 23 ARID1A-deficient carcinomas, $86 \%$ of the nonatypical endometriosis (12 of 14 ) and $100 \%$ of the atypical endometriosis (14 of 14), benign (3 of 3 ), and borderline (6 of 6) clear-cell adenofibroma components were found to be ARID1A deficient. In contrast, in the 19 patients with ARID1A-intact carcinomas, all of the adjacent precursor lesions retained ARID1A expression regardless of their types and cytological atypia. Analysis of 22 solitary endometrioses and 10 endometrioses distant from ARID1A-deficient carcinomas showed that all of these lesions were diffusely immunoreactive for ARID1A. Among the 42 clear-cell carcinomas, somatic mutations of PIK3CA were detected in $17(40 \%)$ tumors and majority $(71 \%)$ of these were ARID1A-deficient carcinomas. These results suggest that loss of ARID1A protein expression occurs as a very early event in ovarian clear-cell carcinoma development, similar to the pattern of PIK3CA mutation recently reported by our group, and frequently coexists (not mutually exclusive) with PIK3CA mutations.
\end{abstract}

Modern Pathology (2012) 25, 615-624; doi:10.1038/modpathol.2011.189; published online 9 December 2011

Keywords: ARID1A; clear-cell adenofibroma; clear-cell carcinoma; endometriosis; PIK3CA

Among ovarian carcinomas, clear-cell carcinoma has been recognized as a distinct clinicopathological

Correspondence: Dr S Yamamoto, MD, Department of Basic Pathology, National Defense Medical College, 3-2 Namiki, Tokorozawa, Saitama 359-8513, Japan.

E-mail: dr21001@ndmc.ac.jp

Received 19 July 2011; revised 5 September 2011; accepted 21 September 2011; published online 9 December 2011 entity in view of its characteristic histology, frequent concurrence of endometriotic lesions, and highly chemoresistant nature, resulting in an extremely poor prognosis when surgical cytoreduction is insufficient. ${ }^{1-4}$ Although little is known about the molecular genetic alterations underlying tumor development, a hypothesis of multistep tumorigenesis of ovarian clear-cell carcinoma, starting with histologically benign-appearing precursor lesions 
(ie, endometriosis and benign clear-cell adenofibroma), progressing to their atypical counterparts (ie, atypical endometriosis and borderline clearcell adenofibroma), and ultimately to clear-cell carcinoma, has been proposed and widely considered. $^{5-11}$ To establish novel therapeutic strategies for ovarian clear-cell carcinoma, it is crucial to elucidate the molecular aberrations that are characteristic of this type of carcinoma during tumor development.

Although little is known about the pathogenesis of ovarian clear-cell carcinoma, somatic mutations of the ARID1A (the AT-rich interactive domain 1A) and PIK3CA genes are the most common molecular genetic alterations identified thus far in ovarian clear-cell carcinomas. ${ }^{12-15}$ ARID1A (also known as BAF250a), a protein encoded by the ARID1A gene located on chromosome 1p36, is a key component of the multi-protein SWI/SNF chromatin-remodeling complex present in all eukaryotes and is believed to confer specificity in the regulation of gene expression. ${ }^{16}$ The chromatin-remodeling activity of SWI/SNF has an integral role in controlling gene expression and is critical in tissue development, cellular differentiation, and tumor suppression. ${ }^{16-20}$ Inactivation of ARID1A is thought to enhance cellcycle progression by potentially involving c-myc, thereby contributing to uncontrolled cellular proliferation in cancer cells. ${ }^{21-23}$ Recently, with the use of whole-exome sequencing and transcriptome sequencing, two independent studies showed novel somatic mutations of the ARID1A gene in 43 and $56 \%$ of ovarian clear-cell carcinomas, suggesting a major role of ARID1A in the pathogenesis of this carcinoma type. ${ }^{12,13}$ However, to date, it is unclear whether the alteration of ARID1A is an early or late event in the development and progression of ovarian clear-cell carcinoma.

The PIK3CA gene, encoding the catalytic subunit p110a of phosphatidylinositol-3 kinases (PI3K), is located on chromosome 3q26.3, and both somatic mutations and gene amplification of PIK3CA increase PI3K activity and activate the downstream Akt signaling pathway. ${ }^{24-26}$ Somatic activating mutations of PIK3CA have been reported in ovarian clear-cell carcinomas with high frequencies of $32-46 \% .{ }^{14,15}$ Our recent study showed that somatic mutations of PIK3CA were also present in the coexisting endometriotic epithelium, adjacent to the PIK3CA-mutation-positive clear-cell carcinomas, even in those lacking cytological atypia. ${ }^{15}$ These findings suggest that mutations of the PIK3CA gene occur before the development of the atypical precancerous lesions (ie, atypical endometriosis) and that PIK3CA mutations are very early events in the development of endometriosisassociated ovarian clear-cell carcinoma. It is therefore of interest to determine whether PIK3CA mutations and ARID1A could coexist or be mutually exclusive in the pathogenesis of ovarian clear-cell carcinoma.
In the present study, the timing of the alterations of ARID1A expression during the development and progression of ovarian clear-cell carcinoma was examined. For this purpose, 42 patients with clear-cell carcinoma belonging to a group of tumors with adjacent putative precursor lesions were selected. As the immunohistochemical analysis of clear-cell and endometrioid carcinomas of the ovary showed that loss of ARID1A protein expression was highly correlated with the presence of ARID1A mutations, ${ }^{12,27}$ and as the ARID1A mutations are randomly distributed in 20 exons and most of these are the insertion/deletion type of mutations, making it very difficult to be detected in formalinfixed paraffin-embedded specimens, ${ }^{12,13}$ we used immunohistochemistry to assess possible alterations of ARID1A. ARID1A expression was specifically examined in the benign-appearing putative precursor lesions (ie, non-atypical endometriosis and benign clear-cell adenofibroma), their atypical forms (atypical endometriosis and borderline clear-cell adenofibroma), and the corresponding invasive carcinoma components. Moreover, to assess the possible relationships between ARID1A and PIK3CA alterations, immunoreactivity for ARID1A was compared with the mutational status of PIK3CA. In ovarian carcinomas, $>80 \%$ of mutations in PIK3CA have been found in the helical (exon 9) and kinase domains (exon 20). ${ }^{14,24,25}$ Therefore, we restricted our analyses to these regions of the gene.

\section{Materials and methods}

\section{Cases and Histological Components Analyzed}

Hematoxylin- and eosin-stained sections from a consecutive series of 90 primary clear-cell adenocarcinoma cases, retrieved from the files of the Department of Laboratory Medicine; National Defense Medical College Hospital; Japan, were histologically reviewed. On the basis of the histopathological criteria described previously, ${ }^{5,7-9}$ of the 90 patients, 28 patients with synchronous endometriotic lesions (endometriosis-associated carcinomas) and 14 patients with adjacent clear-cell adenofibroma components (adenofibroma-associated carcinomas) were identified. All these 42 patients had undergone surgical resection between 1986 and 2007, and none had undergone chemotherapy or radiation therapy before surgery. All specimens analyzed were formalin-fixed and paraffin-embedded tissue sections. There was no overlap between the endometriosis-associated and adenofibroma-associated carcinoma cases. The diseases were clinically staged according to the International Federation of Gynecology and Obstetrics system. Of the 28 patients with endometriosisassociated carcinoma, 18 (64\%) had stage I, 3 $(11 \%)$ had stage II, $5(18 \%)$ had stage III, and 2 $(7 \%)$ had stage IV carcinomas. Of the 14 patients 
with adenofibroma-associated carcinoma, 10 (71\%) had stage I, $1(7 \%)$ had stage II, $2(14 \%)$ had stage III, and $1(7 \%)$ had stage IV carcinoma. Twenty-two solitary endometrioses were also identified from salpingo-oophorectomy specimens. The research protocol was approved by the ethics committee of the National Defense Medical College, Tokorozawa, Japan.

On the basis of the previously described histological criteria for 'atypical endometriosis', $, 7,8$ endometriotic lesions adjacent to clear-cell adenocarcinoma were classified into non-atypical and atypical forms. Of the 28 patients with endometriosis-associated carcinoma, 15 had both nonatypical and atypical endometriosis, 6 had only non-atypical endometriosis, and 7 had only atypical endometriosis. Therefore, 21 non-atypical and 22 atypical endometriotic lesions as well as 28 endometriosis-associated invasive carcinoma components were analyzed by immunohistochemistry.

With reference to the histopathological criteria of benign and borderline clear-cell adenofibroma described previously, ${ }^{7,9}$ adenoma lesions in each adenofibroma were histologically subclassified into benign and borderline categories. Consequently, 9 of the 14 adenofibroma-associated patients had both the evaluable benign and borderline (clear-cell) adenofibroma components, 4 had the borderline adenofibroma component only, and 1 had the benign adenofibroma only. Therefore, 10 benign adenofibroma components, 13 borderline components, and 14 adenofibroma-associated invasive carcinoma components were analyzed by immunohistochemistry.

\section{Immunohistochemistry for ARID1A Detection}

All the selected formalin-fixed and paraffinembedded specimens were cut into $4 \mu \mathrm{m}$ thick serial sections and analyzed by immunohistochemistry. A commercially available polyclonal rabbit antiARID1A antibody (HPA005456; Sigma-Aldrich; diluted 1:50) was used as the primary antibody for ARID1A protein detection, as described previously. ${ }^{28}$ Sections were deparaffinized and boiled in an autoclave at $121^{\circ} \mathrm{C}$ for $15 \mathrm{~min}$ in $0.01 \mathrm{~mol} / \mathrm{l}$ citrate buffer ( $\mathrm{pH}$ 6.0) and then allowed to cool at room temperature. Endogenous peroxidase activity was blocked using $5 \%$ hydrogen peroxide. The slides were incubated at $4{ }^{\circ} \mathrm{C}$ overnight with primary antibodies and then reacted with a dextran polymer reagent combined with secondary antibodies and peroxidase for $1 \mathrm{~h}$ at room temperature. Specific antigen-antibody reactions were visualized with $0.2 \%$ diaminobenzidine tetrahydrochloride and hydrogen peroxide, and counterstaining was performed using Mayer's hematoxylin. Non-neoplastic cells, including endothelial cells, fibroblasts, and lymphocytes, normally show ARID1A nuclear immunoreactivity, and therefore, they served as positive internal controls. As negative controls, sections without the primary antibody were used.

Previous studies have shown that inactivating mutations of ARID1A are associated with the loss of protein expression. ${ }^{12,27}$ Therefore, we focused our attention on the lesions with undetectable ARID1A immunoreactivity in the nucleus and used a scoring system to classify all lesions into ARID1A deficient (immunoreactions undetectable) and ARID1A intact (immunoreactions detectable); any level of ARID1A immunoreactivity was considered ARID1A intact. As the majority of the carcinomas showed an ARID1A immunoreaction that was diffusely ( $>80 \%$ of the cells of interest) immunoreactive or homogeneously undetectable, we did not consider the percentage of the immunoreactive cells in the examined lesions.


Figure 1 ARID1A immunoreactivity in two representative ovarian clear-cell carcinoma cases. (a) A clear-cell carcinoma showing diffuse and strong nuclear immunoreactivity for ARID1A. (b) A clear-cell carcinoma with undetectable ARID1A immunoreactivity. Stromal cells of both the samples show moderate to strong immunoreactivity and serve as internal positive controls. The carcinomas shown in panels a and $\mathbf{b}$ were defined as ARID1A-intact and ARID1A-deficient carcinomas, respectively. Immunoperoxidase stain, original magnification $\times 200$ for (a) and $\times 400$ for (b). 


\section{Mutation Assay of the PIK3CA Gene}

Invasive carcinoma components from the enrolled 42 cases were analyzed for somatic mutations of the PIK3CA gene using direct genomic DNA sequencing. Details of these methods have been described in our previous study. ${ }^{15}$ Briefly, exons 9 and 20 of the PIK $3 C A$ gene were amplified using PCR for genomic DNA. PCR was conducted using AmpliTaq Gold (Applied Biosystems, Foster City, CA). The primers for PCR and sequencing conditions have been described previously. ${ }^{15}$ The PCR products were subsequently subjected to direct sequencing PCR with BigDye terminator V 3.1/1.1 cycle sequencing reagents (Applied Biosystems), and samples were analyzed on an ABI PRISM 3130
Genetic Analyzer (Applied Biosystems) with DNA Sequencing Analysis Software v5.2 (Applied Biosystems). For 23 of the 28 patients with endometriosis-associated carcinomas, we used the required data from our previous study. ${ }^{15}$ The remaining 5 patients with endometriosis-associated carcinomas and 14 with adenofibroma-associated carcinomas were analyzed for the first time in the present study.

\section{Statistical Analysis}

Statistical analyses were performed using StatMate III software (ATMS, Tokyo, Japan). Comparisons between parameters were computed using the $\chi^{2}$ test or Student's $t$-test for unpaired data.
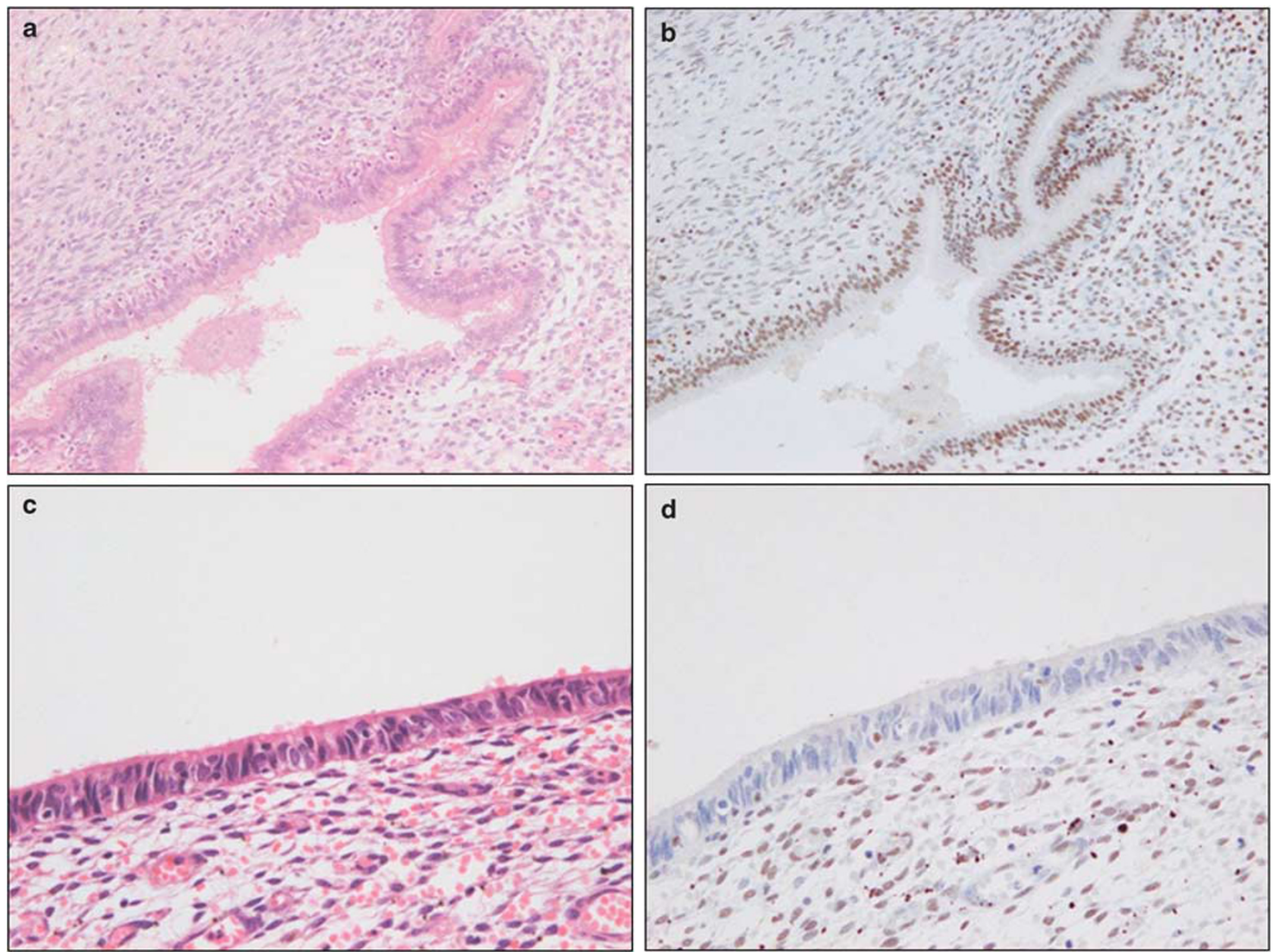

Figure 2 Representative histological features and ARID1A immunoreactions in solitary endometriosis (a, b), non-atypical endometriosis adjacent to clear-cell carcinoma (c, d), and atypical endometriosis adjacent to clear-cell carcinoma (e-h). (a) Hematoxylin and eosin (HE) staining of a solitary endometriosis sample. (b) Epithelial cells and stromal cells show diffusely immunoreactive for ARID1A. (c) A case of non-atypical endometriosis lacking evident cytological or structural atypia in the epithelial cells. (d) Stromal cells are diffusely immunoreactive for ARID1A, while the immunoreaction is undetectable in the endometriotic epithelia. (e) A case of atypical endometriosis showing moderate degrees of cytological atypia and cellular stratification. (f) Similar to panel d, the ARID1A immunoreaction is undetectable in epithelia. (g) HE staining of another case of atypical endometriosis. (h) ARID1A immunostaining of the samples shown in (g). Epithelial cells, as well as stromal cells, show diffuse immunoreactivity for ARID1A. (a, c, e, and g) HE stain, original magnification $\times 200$ for $(\mathbf{a}, \mathbf{e}$, and $\mathbf{g})$ and $\times 400$ for $(\mathbf{c})$. (b, d, f, and $\mathbf{h})$ Immunoperoxidase stain, original magnification $\times 200$ for $(\mathbf{b}, \mathbf{f}$, and $\mathbf{h})$ and $\times 400$ for $(\mathbf{d})$. 



Figure 2 Continued.

Values of $P<0.05$ were considered to be statistically significant.

\section{Results}

\section{ARID1A Expression in Invasive Carcinoma Components}

Analysis of invasive carcinoma components showed that immunoreactivity was always in a diffusely (not heterogeneously) detectable or homogeneously undetectable pattern. Of the 28 endometriosis-associated carcinomas, $17(61 \%)$ and $11(39 \%)$ were judged as the ARID1A-deficient and the ARID1Aintact tumors, respectively (Figure 1). Of the 14 adenofibroma-associated carcinomas, 6 (43\%) and $8(57 \%)$ were judged as the ARID1A-deficient and the ARID1A-intact tumors, respectively. Overall, 23 $(55 \%)$ of the 42 clear-cell carcinomas analyzed were determined as ARID1A deficient. Comparison between the endometriosis-associated carcinomas and adenofibroma-associated carcinomas showed no statistically significant differences in the frequency of deficiency of ARID1A immunoreaction $(P=0.273)$.

\section{ARID1A Expression in Endometriosis}

All of the 22 solitary endometriosis samples showed diffuse and strong immunoreactions for ARID1A (Figures 2a and b; Table 1).

Among the 17 patients with endometriosis-associated carcinomas that were ARID1A-deficient tumors, 14 non-atypical endometrioses and 14 atypical endometrioses were histologically identified. Of the 14 non-atypical endometrioses, 12 (86\%) and $2(14 \%)$ endometrioses were judged as ARID1A deficient and ARID1A intact, respectively (Figures 2C and d), and all of the 14 atypical endometrioses were ARID1A deficient (Figures 2e and f). Three (25\%) of the 12 ARID1A-deficient non-atypical endometrioses showed front formation during immunoreactivity, ie, the areas of ARID1A-deficient cells were adjacent to the ARID1A-intact areas, giving the impression of an 'abrupt loss' of ARID1A expression (Figure 3).

Among the 11 endometriosis-associated carcinoma cases with an ARID1A-intact carcinoma, 7 non-atypical and 8 atypical endometrioses lesions were identified, and all 15 lesions showed diffusely immunoreactivity for ARID1A (Figures $2 g$ and $h$ ). 
Table 1 The ARID1A immunoreactivity in the putative precursor lesions of ovarian clear-cell carcinomas

\begin{tabular}{|c|c|c|}
\hline & \multicolumn{2}{|c|}{$\begin{array}{l}\text { Number of } \\
\text { cases }(\%)\end{array}$} \\
\hline & ARID1A & ARID1A \\
\hline Type of precursor lesion & Deficient & Intact \\
\hline \multicolumn{3}{|l|}{ In the endometriosis-associated carcinomas } \\
\hline $\begin{array}{l}\text { Non-atypical endometriosis } \\
\text { (distant; } n=10 \text { ) }\end{array}$ & 0 & $10(100)$ \\
\hline $\begin{array}{l}\text { Non-atypical endometriosis } \\
\text { (adjacent; } n=14 \text { ) }\end{array}$ & $12(86)^{\mathrm{a}}$ & $2(14)$ \\
\hline $\begin{array}{l}\text { Atypical endometriosis } \\
\text { (adjacent; } n=14 \text { ) }\end{array}$ & $14(100)$ & 0 \\
\hline \multicolumn{3}{|l|}{ In the ARID1A-intact carcinomas } \\
\hline $\begin{array}{l}\text { Non-atypical endometriosis } \\
\text { (adjacent; } n=7 \text { ) }\end{array}$ & 0 & $7(100)$ \\
\hline Atypical endometriosis $(n=8)$ & 0 & $8(100)$ \\
\hline \multicolumn{3}{|l|}{ In the adenofibroma-associated carcinomas } \\
\hline $\begin{array}{l}\text { Benign clear-cell adenofibroma } \\
\text { (adjacent; } n=3 \text { ) }\end{array}$ & $3(100)$ & 0 \\
\hline $\begin{array}{l}\text { Borderline clear-cell adenofibroma } \\
\text { (adjacent; } n=6 \text { ) }\end{array}$ & $6(100)$ & 0 \\
\hline \multicolumn{3}{|l|}{ In the ARID1A-intact carcinomas } \\
\hline $\begin{array}{l}\text { Benign clear-cell adenofibroma } \\
\text { (adjacent; } n=10 \text { ) }\end{array}$ & 0 & $7(100)$ \\
\hline $\begin{array}{l}\text { Borderline clear-cell adenofibroma } \\
\text { (adjacent; } n=14 \text { ) }\end{array}$ & 0 & $7(100)$ \\
\hline
\end{tabular}

${ }^{\mathrm{a}}$ Three of these 12 lesions exhibited a pattern of immunoreactivity in which areas of ARID1A-deficient cells were adjacent to the ARID1Aintact areas.

Of the 23 patients with endometriosis-associated carcinomas that were ARID1A deficient, 10 lesions of non-atypical endometriosis distant from carcinoma components were also identified, and all 10 lesions were diffusely immunoreactive for ARID1A.

Consequently, with the exception of three ARID1Adeficient non-atypical endometrioses showing heterogeneous immunoreaction (area of ARID1Adeficient cells was adjacent to the area of ARID1Aintact cells), all of the endometriotic lesions analyzed showed diffusely detectable or homogeneously undetectable immunoreaction patterns for ARID1A.

\section{ARID1A Expression in Clear-Cell Adenofibroma}

Among the six cases of adenofibroma-associated carcinomas that were ARID1A-deficient tumors, three benign clear-cell adenofibroma and six clear-cell borderline adenofibroma components were histologically identified (Table 1). All three benign adenofibromas and six borderline adenofibroma components were judged as ARID1A deficient (Figures 4a-d).

Among the eight cases of adenofibroma-associated carcinomas that were ARID1A-intact tumors, seven benign adenofibromas and seven borderline adeno-
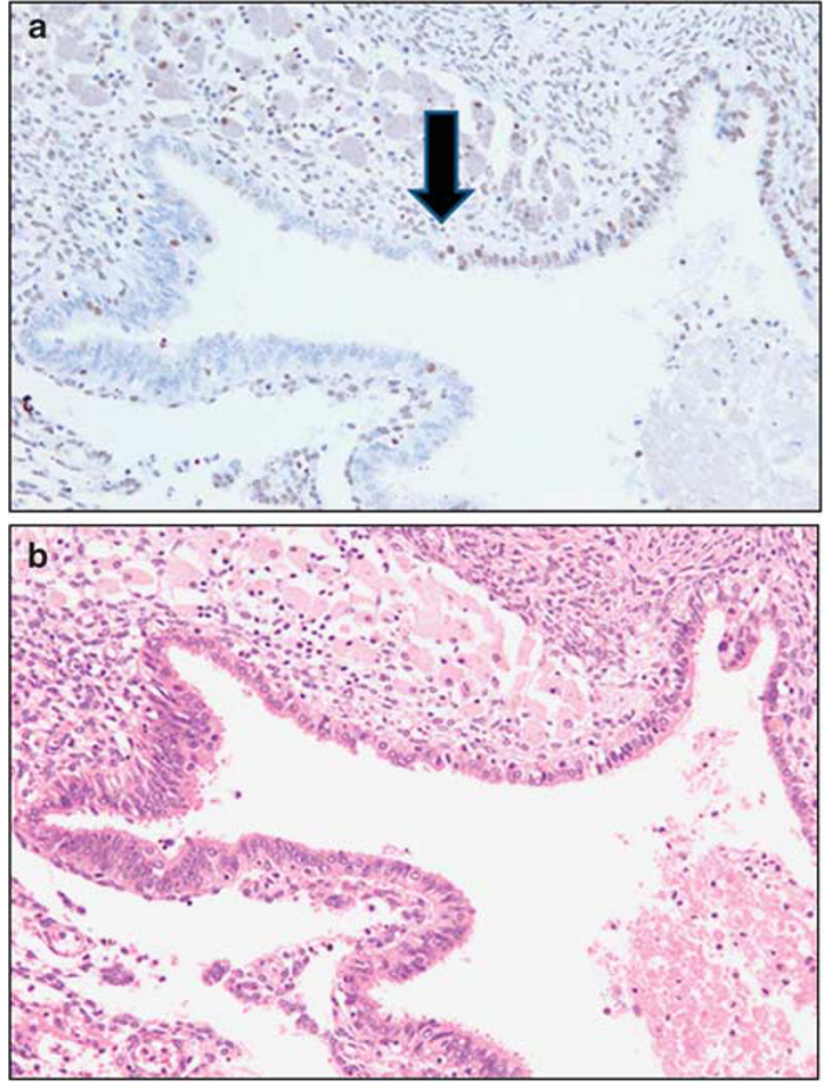

Figure 3 Representative case of a non-atypical endometriosis lesion adjacent to clear-cell carcinoma, showing a pattern of heterogeneous immunoreactions for ARID1A. (a) The area of ARID1A-deficient cells is adjacent to the ARID1A-intact area and shows front formation, namely the 'abrupt loss of ARID1A expression' (arrow), suggesting that mutations arose in clones within this lesion. (b) Hematoxylin and eosin (HE) staining corresponding to the samples shown in panel a. Regarding the histomorphology or the extent of cellular atypia, the ARID1Aintact and ARID1A-deficient epithelia show similar appearance. Original magnification, $\times 200$ each.

fibroma components were histologically identified, and all 14 lesions were diffusely immunoreactive for ARID1A (Figures 4e and f).

Consequently, all the clear-cell adenofibroma components analyzed showed a diffusely detectable or homogeneously undetectable immunoreaction pattern for ARID1A.

\section{Relationship Between ARID1A Immunoreactivity and PIK3CA Mutations}

Sequencing chromatograms for PIK3CA exons 9 and 20 were obtained for all the 42 carcinomas analyzed. Direct sequencing showed mutations in $17(40 \%)$ carcinomas: $14(83 \%)$ were found in exon 20 (all of these were H1047R substitutions); and the remaining $3(17 \%)$ were in exon 9 (two were E545K substitutions and one was an E545V substitution). Of the 17 PIK3CA-mutation-positive carcinomas, 12 $(71 \%)$ tumors were ARID1A-deficient carcinomas; 

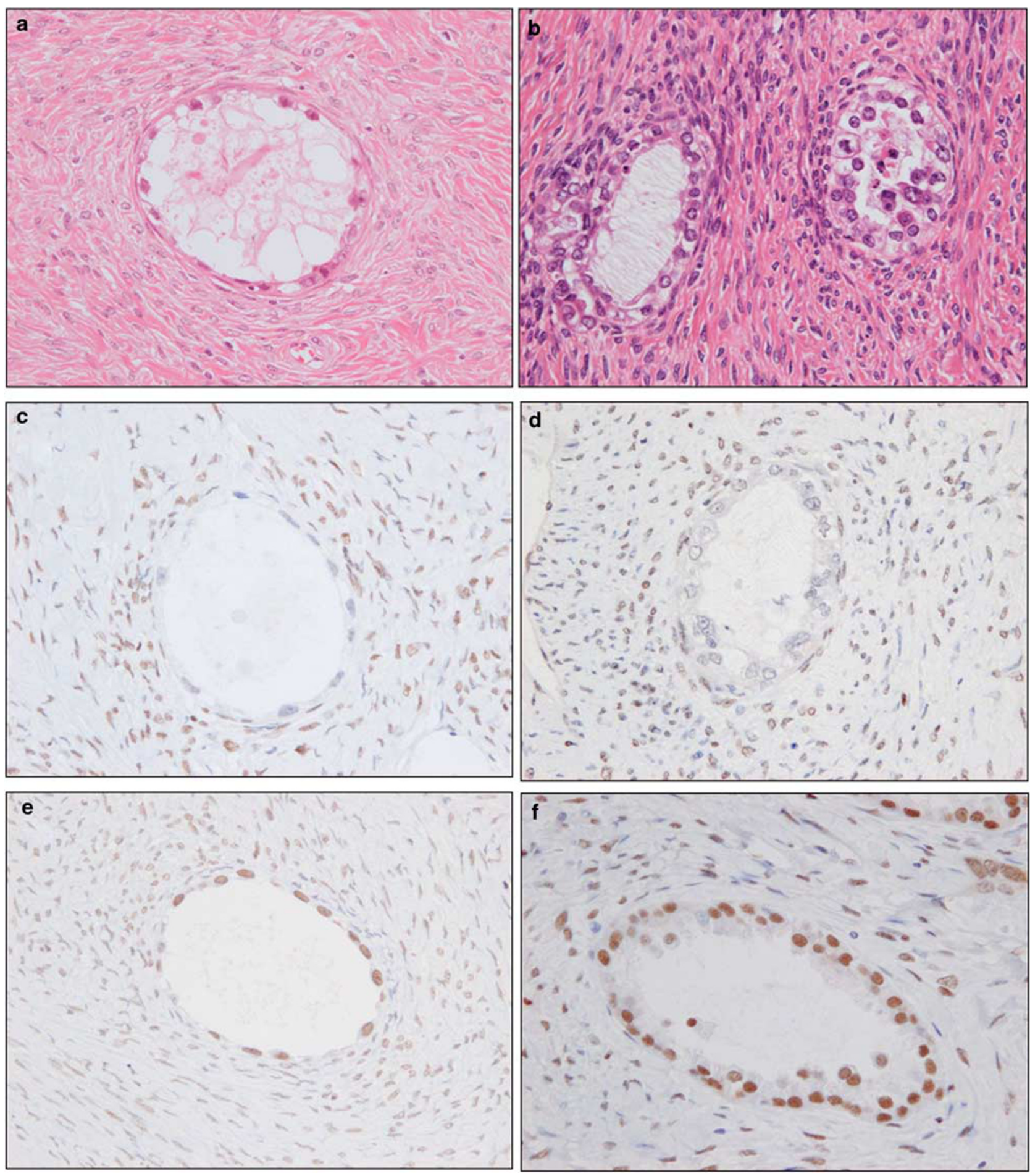

Figure 4 Representative histological features and ARID1A immunoreactions in benign clear-cell adenofibroma (a, c, and e) and borderline clear-cell adenofibroma (b, d, and f). (a) A case of benign clear-cell adenofibroma lacking cytological or structural atypia. (b) A case of borderline clear-cell adenofibroma. Epithelial cells show a moderate degree of cytological atypia. (c, d) Stromal cells are diffusely immunoreactive for ARID1A, while the immunoreaction is undetectable in epithelial cells. (e, f) Epithelial cells, as well as stromal cells, show diffuse immunoreactivity for ARID1A. (a, b) Hematoxylin and eosin (HE) staining, original magnification $\times 400$ each. $(\mathbf{c}-\mathbf{f})$ Immunoperoxidase stain, original magnification $\times 400$ each.

of the 19 ARID1A-intact carcinomas, PIK3CA mutations were detected in only $5(26 \%)$ tumors (Table 2). The frequency of PIK3CA mutations in the
ARID1A-deficient carcinomas was higher than that in the ARID1A-intact carcinomas, but not significantly different $(P=0.083)$. 
The ten endometriosis-associated carcinoma cases, in which our previous study analyzed the status of PIK3CA mutations both in clear-cell carcinoma and coexisting endometriotic lesions, ${ }^{15}$ were combined with the data obtained from the present study (Table 3). Of these 10 carcinoma components harboring PIK3CA mutations, all but one carcinomas (case no. 12) were ARID1A deficient. All of the six PIK3CA-mutation-positive nonatypical endometrioses (case no. 5, 8, 14, 17, 18, and 20) were immunohistochemically ARID1A deficient. Moreover, except for case no. 12, all of the six atypical endometrioses harboring PIK3CA mutations were of ARID1A deficient. In case no. 6, PIK3CA mutation was firstly documented in the invasive carcinoma component, but ARID1A immunoreactions was already deficient in the adjacent atypical endometriosis.

\section{Discussion}

In recent years, genome-wide sequencing analyses have made substantial progress in cataloging molecular genetic alterations in human cancers. One of the most significant recent findings is the identification of somatic mutations of several chromatinremodeling genes in certain tumor types. These

Table 2 Comparison between the ARID1A immunoreactivity and PIK3CA mutations in ovarian clear-cell carcinomas

\begin{tabular}{lccc}
\hline PIK3CA mutations & \multicolumn{2}{c}{ Number of cases (\%) } & \multirow{2}{*}{ P-value } \\
\cline { 2 - 3 } & ARID1A & ARID1A & \\
\hline In exon 9 or 20 & Deficient & Intact & \\
Present $(n=17)$ & $12(71)$ & $5(29)$ & 0.083 \\
Absent $(n=25)$ & $11(44)$ & $14(56)$ & \\
\hline
\end{tabular}

genes include the JARID1C gene in renal cell carcinomas, ${ }^{29}$ SMARCA4/BRG1 (SWI/SNF-related, matrix-associated, actin-dependent regulator of chromatin, subfamily a, member 4) in lung carcinomas, ${ }^{30,31}$ and most recently, ARID1A in ovarian and endometrial cancers. ${ }^{12,13,28}$ The mutations in ARI$D 1 A$ were widely distributed throughout the coding region and all were predicted to truncate the protein through a base substitution, resulting in a stop codon or an out-of-frame insertion or deletion. These alterations resulted in the loss of ARID1A protein expression, suggesting that somatic mutations inactivate the gene product as a tumor suppressor. ${ }^{12,13}$

The main findings of our investigation can be summarized as follows: (a) loss of ARID1A expression in the putative precursor lesions of ovarian clear-cell carcinoma occurred specifically in cases in which the corresponding carcinoma was ARID1A deficient. (b) In majority of the ARID1A-deficient carcinoma cases, the deficiency of ARID1A immunoreaction was already evident at the stage of precursor lesions lacking cytological atypia (ie, non-atypical endometriosis adjacent to carcinoma or benign clear-cell adenofibroma), suggesting that loss of ARID1A protein occurs as a very early event in tumorigenesis. (c) Even in patients with ARID1Adeficient clear-cell carcinoma, endometriosis distant from the carcinoma was always ARID1A intact. (d) Loss of ARID1A expression and PIK3CA mutations frequently coexisted and were not mutually exclusive. Although in the current study the mutational status of the ARID1A gene was not known, in ovarian clear-cell carcinomas, the presence of ARID1A mutations were adequately, but not perfectly, correlated with the loss of ARIDA1 immunoreactivity. ${ }^{12,27}$ Therefore, the data obtained in the present study may indicate how early ARID1A is mutated during the development of ovarian clearcell carcinoma.

Table 3 Frequent concurrence of PIK3CA mutations and loss of ARID1A immunoreactivity in the endometriosis-associated ovarian clear-cell carcinoma development

Case no.

(patient's age (years))
Status of PIK3CA/immunoreaction for ARID1A

\begin{tabular}{|c|c|c|}
\hline $\begin{array}{l}\text { Non-atypical } \\
\text { endometriosis }\end{array}$ & $\begin{array}{l}\text { Atypical } \\
\text { endometriosis }\end{array}$ & $\begin{array}{l}\text { Clear-cell } \\
\text { carcinoma }\end{array}$ \\
\hline Mut/deficient & Mut/deficient & Mut/deficient \\
\hline Wt/intact & Wt/deficient & Mut/deficient \\
\hline Mut/deficient & $-1-$ & Mut/deficient \\
\hline Wt/intact & Mut/intact & Mut/intact \\
\hline$-1-$ & Mut/deficient & Mut/deficient \\
\hline Mut/deficient & Mut/deficient & Mut/deficient \\
\hline$-1-$ & Mut/deficient & Mut/deficient \\
\hline Mut/deficient & Mut/deficient & Mut/deficient \\
\hline Mut/deficient & Mut/deficient & Mut/deficient \\
\hline Mut/deficient & $-1-$ & Mut/deficient \\
\hline
\end{tabular}

${ }^{\mathrm{a}}$ Clinical stages of disease defined by the International Federation of Gynecology and Obstetrics.

Mut, somatic mutations (+); Wt, wild type for exon 9 and 20; $-/-$, corresponding components were not histologically identified.

The data of this study combined with those obtained from our previous study. ${ }^{15}$ 
Several recent studies have strongly indicated that loss of ARID1A protein expression and/or its mutations has an important role in the pathogenesis of certain ovarian cancers thought to be derived from endometriosis. ${ }^{12,13,28}$ However, in the present study, in $43 \%$ (6 of 14) of the cases, loss of ARID1A expression was also associated with another putative ovarian clear-cell carcinogenic pathway, namely, the adenofibroma-associated pathway. These results suggest that regardless of the pathogenic pathway involved (ie, endometriosis-associated vs adenofibroma-associated pathways), ARID1A alterations might be commonly associated with the development of ovarian clear-cell carcinoma.

In the present study, excluding the cases of nonatypical endometriosis adjacent to the ARID1Adeficient carcinomas, all lesions analyzed showed diffusely detectable or homogenously undetectable ARID1A immunoreactivity. These findings contrast those of the study by Guan et $a l^{28}$ in which a fraction of uterine endometrioid carcinomas with ARID1A mutations were characterized by a heterogeneous immunostaining pattern for ARID1A. However, this type of heterogeneous loss of ARID1A immunoreactions was only detected in tumors with $A R I D 1 A$ mutations and not in those without mutations, ${ }^{28}$ suggesting that $A R I D 1 A$ mutations occurred in the subclones of cells within these endometrial carcinomas. Wiegand et $a l^{32}$ reported that a loss of ARID1A protein expression was not detected in any of the nine lesions of endometrial complex atypical hyperplasia considered as putative precursors of uterine endometrioid carcinoma. On the basis of the complete data available, it is likely that (1) ARID1A mutations occur as very early events in ovarian clear-cell carcinoma development, creating ARID1A-deficient subclones of a neoplastic nature in the endometriosis tissues or facilitating tumor initiation in clear-cell adenofibroma and (2) the biological significance or timing of occurrence of alterations of ARID1A expression might differ between ovarian clear-cell carcinoma and endometrial carcinoma; in the latter, ARID1A alterations may be a late event in tumorigenesis. Moreover, the present analysis showed that, in the three nonatypical endometrioses that showed a pattern of heterogeneous ARID1A immunoreactivity, areas of ARID1A-deficient cells were detected adjacent to those of the ARID1A-intact areas, suggesting that mutations might arise in clones within these lesions.

Another interesting finding of the present study was the frequent coexistence of ARID1A alterations with the PIK3CA gene mutations. Guan et $a l^{28}$ showed that the loss of ARID1A immunoreactivity and ARID1A somatic mutations were frequently (26 and $40 \%$, respectively) detected in uterine endometrioid carcinoma in comparison with other histological subtypes of uterine carcinomas. Endometrioid carcinoma of the uterus frequently harbors sequence mutations in PIK3CA (estimated 24-39\% of cases) as well as in the CCNB1 and PTEN genes, and these mutations are rarely found in uterine serous carcinoma. ${ }^{33-36}$ Moreover, in a previous comparative study of 44 endometrioid adenocarcinomas and 29 precursor lesions (complex atypical hyperplasias), PIK3CA mutations were identified in $39 \%$ of the former but only $7 \%$ of the latter, suggesting that such mutations represent a late event in endometrial carcinogenesis, which is similar to the data on ARIDA1 alterations in this type of tumor. ${ }^{32,36}$ In contrast to endometrial carcinomas, PIK3CA mutations can occur as an early event in ovarian clear-cell carcinoma development, usually appearing at the stage of non-atypical endometriosis. ${ }^{15}$ Moreover, in the present data combined with our previous study, ${ }^{15}$ concurrence of PIK3CA mutations and loss of ARID1A immunoreactions occurred at the very early stage of the endometriosis-associated clear-cell carcinoma development (ie, at the non-atypical endometriosis). These findings suggest the possibility of cross-talk between the ARID1A and PI3K signaling pathways. Further investigation of this possible cross-talk and assessment of the contribution of ARID1A inactivation to tumor initiation and progression in these tumors should be conducted. Somatic mutations of tumor suppressor genes such as ARID1A could directly lead to epigenetic changes in tumor cells through specific modifications of chromatin proteins. Therefore, by altering the accessibility of transcription factors to chromatin, additional mutations in the PI3 kinase pathway (ie, PIK3CA mutation) would promote its oncogenic properties.

In conclusion, loss of ARID1A protein expression (and possible ARID1A mutations) appears to be an early molecular event in ovarian clear-cell carcinogenesis, regardless of the carcinogenic pathway involved. Moreover, the coexistence of the loss of ARID1A expression and PIK3CA mutations suggested a possible cross-talk between the regulation of ARID1A expression and the PI3K signaling pathway during ovarian clear-cell carcinoma development. An increased understanding of the molecular events involved in the initiation and development of ovarian clear-cell carcinoma will provide a basis for developing novel forms of early diagnosis and therapy.

\section{Acknowledgements}

This work was supported in part by a Grant-in-aid for promotion of defense medicine from the Ministry of Defense, Japan (SY, OM), by a Grant-in-aid for cancer research from the Ministry of Health, Labor, and Welfare, Japan (HT), and by a Grant from the Foundation for Promotion of Cancer Research (SY and HT). We are grateful to Ms Kozue Suzuki, Department of Basic Pathology, National Defense Medical College, Saitama, Japan, for technical assistance. 


\section{Disclosure/conflict of interest}

The authors declare no conflict of interest.

\section{References}

1 Lee KR, Tavassoli FA, Part J, et al. Surface epithelialstromal tumours. In: Tavassoli FA, Devilee P (eds). World Health Organization Classification of Tumours. Pathology and Genetics of Tumours of the Breast and Female Genital Organs. IARC Press: Lyon, 2003, pp 117-145.

2 Chan JK, Teoh D, Hu JM, et al. Do clear cell ovarian carcinomas have poorer prognosis compared to other epithelial cell types? A study of 1411 clear cell ovarian cancers. Gynecol Oncol 2008;109:370-376.

3 Mizuno M, Kikkawa F, Shibata K, et al. Long-term follow-up and prognostic factor analysis in clear cell adenocarcinoma of the ovary. J Surg Oncol 2006;94:138-143.

4 Takano M, Kikuchi Y, Yaegashi N, et al. Clear cell carcinoma of the ovary: a retrospective multicentre experience of 254 patients with complete surgical staging. Br J Cancer 2006;94:1369-1374.

5 Fukunaga M, Nomura K, Ishikawa E, et al. Ovarian atypical endometriosis: its close association with malignant epithelial tumours. Histopathology 1997;30: 249-255.

6 Prowse AH, Manek S, Varma R, et al. Molecular genetic evidence that endometriosis is a precursor of ovarian cancer. Int J Cancer 2006;119:556-562.

7 Yamamoto S, Tsuda H, Takano M, et al. Expression of platelet-derived growth factors and their receptors in ovarian clear-cell carcinoma and its putative precursors. Mod Pathol 2008;21:115-124.

8 Yamamoto S, Tsuda $\mathrm{H}$, Miyai $\mathrm{K}$, et al. Cumulative alterations of p27-related cell-cycle regulators in the development of endometriosis-associated ovarian clear cell adenocarcinoma. Histopathology 2010;56:740-749.

9 Yamamoto S, Tsuda $\mathrm{H}$, Takano $\mathrm{M}$, et al. Clear-cell adenofibroma can be a clonal precursor for clear-cell adenocarcinoma of the ovary: a possible alternative ovarian clear-cell carcinogenic pathway. J Pathol 2008;216:103-110.

10 Veras E, Mao TL, Ayhan A, et al. Cystic and adenofibromatous clear cell carcinomas of the ovary: distinctive tumors that differ in their pathogenesis and behavior: a clinicopathologic analysis of 122 cases. Am J Surg Pathol 2009;33:844-853.

11 Kurman RJ, Shih IeM. The origin and pathogenesis of epithelial ovarian cancer: a proposed unifying theory. Am J Surg Pathol 2010;34:433-443.

12 Wiegand KC, Shah SP, Al-Agha OM, et al. ARID1A mutations in endometriosis-associated ovarian carcinomas. N Engl J Med 2010;363:1532-1543.

13 Jones S, Wang TL, Shih I, et al. Frequent mutations of chromatin remodeling gene ARID1A in ovarian clear cell carcinoma. Science 2010;330:228-231.

14 Kuo KT, Mao TL, Jones S, et al. Frequent activating mutations of PIK3CA in ovarian clear cell carcinoma. Am J Pathol 2009;174:1597-1601.

15 Yamamoto S, Tsuda $\mathrm{H}$, Takano $\mathrm{M}$, et al. PIK3CA mutation is an early event in the development of endometriosis-associated ovarian clear cell adenocarcinoma. J Pathol 2011;225:189-194.

16 Reisman D, Glaros S, Thompson EA. The SWI/SNF complex and cancer. Oncogene 2009;28:1653-1668.
17 Sif S, Saurin AJ, Imbalzano AN, et al. Purification and characterization of mSin3A-containing Brg1 and hBrm chromatin remodeling complexes. Genes Dev 2001;15: 603-618.

18 Wang W, Xue Y, Zhou S, et al. Diversity and specialization of mammalian SWI/SNF complexes. Genes Dev 1996;10:2117-2130.

19 Ho L, Crabtree GR. Chromatin remodelling during development. Nature 2010;463:474-484.

20 Gao X, Tate P, Hu P, et al. ES cell pluripotency and germ-layer formation require the SWI/SNF chromatin remodeling component BAF250a. Proc Natl Acad Sci USA 2008;105:6656-6661.

21 Huang J, Zhao YL, Li Y, et al. Genomic and functional evidence for an ARID1A tumour suppressor role. Genes Chromosomes Cancer 2007;46:745-750.

22 Nagl Jr NG, Patsialou A, Haines DS, et al. The p270 (ARID1A/SMARCF1) subunit of mammalian SWI/ SNF-related complexes is essential for normal cell cycle arrest. Cancer Res 2005;65:9236-9244.

23 Nagl Jr NG, Zweitzig DR, Thimmapaya B, et al. The c-myc gene is a direct target of mammalian SWI/SNFrelated complexes during differentiation-associated cell cycle arrest. Cancer Res 2006;66:1289-1293.

24 Shayesteh L, Lu Y, Kuo WL, et al. PIK3CA is implicated as an oncogene in ovarian cancer. Nat Genet 1999;21:99-102.

25 Samuels Y, Wang Z, Bardelli A, et al. High frequency of mutations of the PIK3CA gene in human cancers. Science 2004;304:554.

26 Kang S, Bader AG, Vogt PK. Phosphatidylinositol 3-kinase mutations identified in human cancer are oncogenic. Proc Natl Acad Sci USA 2005;102:802-807.

27 Maeda D, Mao TL, Fukayama M, et al. Clinicopathological significance of loss of ARID1A immunoreactivity in ovarian clear cell carcinoma. Int J Mol Sci 2010;11:5120-5128.

28 Guan B, Mao TL, Panuganti PK, et al. Mutation and loss of expression of ARID1A in uterine low-grade endometrioid carcinoma. Am J Surg Pathol 2011;35: 625-632.

29 Dalgliesh GL, Furge K, Greenman C, et al. Systematic sequencing of renal carcinoma reveals inactivation of histone modifying genes. Nature 2010;463:360-363.

30 Medina PP, Romero OA, Kohno T, et al. Frequent BRG1/SMARCA4-inactivating mutations in human lung cancer cell lines. Hum Mutat 2008;29:617-622.

31 Rodriguez-Nieto S, Canada A, Pros E, et al. Massive parallel DNA pyrosequencing analysis of the tumor suppressor BRG1/SMARCA4 in lung primary tumors. Hum Mutat 2010;32:E1999-E2017.

32 Wiegand KC, Lee AF, Al-Agha OM, et al. Loss of BAF250a (ARID1A) is frequent in high-grade endometrial carcinomas. J Pathol 2011;224:328-333.

33 Di Cristofano A, Ellenson LH. Endometrial carcinoma. Annu Rev Pathol 2007;2:57-85.

34 Oda K, Stokoe D, Taketani Y, et al. High frequency of coexistent mutations of PIK3CA and PTEN genes in endometrial carcinoma. Cancer Res 2005;65:10669-10673.

35 Velasco A, Bussaglia E, Pallares J, et al. PIK3CA gene mutations in endometrial carcinoma. Correlation with PTEN and K-Ras alterations. Hum Pathol 2006;37:1465-1472.

36 Hayes MP, Wang H, Espinal-Witter R, et al. PIK3CA and PTEN mutations in uterine endometrioid carcinoma and complex atypical hyperplasia. Clin Cancer Res 2006;12:5932-5935. 